\title{
Diagnostic Performance Characteristics of Rapid Dipstick Test for Plasmodium vivax Malaria
}

\author{
Gonul Aslan/ ${ }^{+}$, Mustafa Ulukanligil, Adnan Seyrek, Ozcan Erel*
}

\begin{abstract}
Research Hospital, Department of Microbiology and Parasitology, Medical Faculty, Mersin University, 33070
Zeytinlibahce, Mersin, Turkey *Department Biochemistry, Medical Faculty, Harran University, Şanlıurfa,

Turkey
\end{abstract}

We compared the diagnostic performance characteristics of newly developed method, the rapid dipstick test, which provides colorimetric determination by developing antibody to the lactate dehydrogenase enzyme of parasites, with conventional standard thick-blood film examination. For the rapid test, OptiMAL commercial kits were used. The results were also evaluated with clinical findings from patients. The parasites were determined by microscopic examination of thick-blood films from 81 patients with vivax malaria from southeastern Anatolia, Turkey. The OptiMAL test results were found to be negative in five patients who were diagnosed clinically and through thick-film testing as having vivax malaria. There was no false positivity observed with the OptiMAL test. We concluded that this rapid malaria test has a lower level of sensitivity than the classical thick-blood-film test for malaria, but that these methods have equal specificity.

Key words: malaria - Plasmodium vivax - parasite lactate dehydrogenase (pLDH) - OptiMAL dipstick test

More than 3 billion people worldwide live in malarious regions. Every year, 200 million people are infected with malaria and 2 million of these die from the disease. There are four species causing human malaria: Plasmodium falciparum, $P$. vivax, $P$. ovale and $P$. malaria. Ninety-five percent of all human malaria is caused by $P$. falciparum and $P$. vivax. The bite of the Anopheles mosquito transmits the malaria parasite to humans (Tirasophon et al. 1994). The cyclic recurrence of malaria epidemics has a tremendous impact on the health infrastructure in developing countries and has an adverse effect on local economies, since infected individuals are often too debilitated to work (Krogstad 1996). The classical gold-standard method for detection of the malaria parasite is the examination of Giemsa-stained thick and thin blood films. However, rapid malaria tests have recently been introduced as alternative methods to conventional microscopy for the diagnosis of malaria (Parra et al. 1991).

Two fast and simple immunochromatographic tests (ICT) based on the dipstick principle have recently become available for the diagnosis of $P$. vivax malaria. Both tests detect circulating para-

${ }^{+}$Corresponding author. Fax: +90-324-337.4305. E-mail: gaslantr@yahoo.com

Received 19 July 2000

Accepted 22 January 2001 site antigen by the use of specific antibodies which are bound to the membrane: ICT Malaria P.f./P.v. (ICT Diagnostics, Sydney, Australia) targets histidine-rich protein 2 (HRP-2) of $P$. vivax and $P$. falciparum (first reported by Garcia et al. 1996), whereas OptiMAL (Flow, Inc., Portland, Oregon) detects parasite-specific lactate dehydrogenase (pLDH) first reported by Palmer et collegues (Garcia et al. 1996, Palmer et al. 1998).

While work has been done on the diagnostic performance of the HRP-2, which is used in the diagnosis of vivax malaria in our region, the diagnosis performance of the pLDH method has not, to our knowledge, been examined. Thus, the aim of the present study was to assess the diagnostic performance characteristics of the OptiMAL test with vivax malaria patients according to clinical findings and the results of classical thick-bloodfilm testing.

\section{MATERIALS AND METHODS}

This study was conducted in Sanliurfa in the southeastern Anatolia region of Turkey during the period September-November 1999. Permission for testing was obtained from patients who were admitted to the Siverek Malaria Eradication Centre. The majority of the blood samples were collected from individuals living in rural areas. A total of 190 whole-blood samples were obtained from patients with malaria-like symptoms, including fever and/or chills, of several days' duration. A completed questionnaire was obtained from each patients. 
Two milliliters of venous blood was drawn into EDTA-coated syringes, distributed into sterile test tubes, and placed immediately on ice. Thin and thick-smear blood films were prepared on the site at the time of specimen collection. The whole-blood samples were stored at $=80^{\circ} \mathrm{C}$ until study.

Microscopic determination of the parasite, and counting the parasitaemia - All the blood films were stained with Giemsa and were initially examined at the Siverek Malaria Eradication Centre by a malaria expert. They were then reexamined independently by two skilled microscopists at the Clinical Microbiology Laboratory of Harran University. A slide was considered to be negative when no parasites were detected in 200 WBCs by either of the microscopists (Beadle et al. 1994).

Parasite density (parasites/ $\mu$ l blood) was calculated for each positive thick film by assuming $8000 \mathrm{WBCs} / \mu \mathrm{l}$ blood. Thus, parasites in thick-film fields were counted until 200 WBCs had been observed, and the parasite count was then multiplied by 40 to give the number of parasites per milliliter of blood. This method does not take into account the known loss of parasites in the thick film, which suggests that it produces an underestimate, but the "standard" white blood cell value is probably too high, which helps to cancel out this error (Warhurst \& Williams 1996).

Malaria diagnosis with OptiMAL assay - The whole-blood samples were tested with the OptiMAL ${ }^{\circledR}$ assay. This utilizes a dipstick coated with monoclonal antibodies against the intracellular metabolic enzyme pLDH. The pLDH antigen is present in parasite-infected erythrocytes and is released from them.

The differentiation of malaria parasites is based on antigenic differences between the pLDH isoforms. Since pLDH is produced only by live plasmodium parasites, this test has the ability to differentiate living organisms from dead one. The test uses two reagents (A and $\mathrm{B}$ ) included in the kit. One drop of blood was mixed with two drops of reagent $\mathrm{A}$, and the sample was allowed to migrate to the top of the dipstick. After $10 \mathrm{~min}$, the strip was cleared by adding two drops of reagents $B$. The appearance of a dark band on the strip indicates a positive reaction for any one of the four major malaria species infecting humans. The monoclonal antibody attached at this area of the strip is against an enzyme common to the four plasmodium species targeted. All stages of the parasite are present in the blood during $P$. vivax infections and the pLDH enzyme is present in all these stages during $P$. vivax infections.

\section{RESULTS}

Eighty-one of the 190 patients who had malaria-like symptoms were diagnosed as having $P$. vivax malaria on the basis of thick-film and clinical findings. Seventy-six of these patients were positive according to the OptiMAL test. Of the five false negative results with the OptiMAL test, four exhibited 1-500 parasites/ $\mu l$, and one appeared to contain 2000 parasites/ $\mu$ l. However, no false positive results with the OptiMAL test were determined. Three of the five false negative patients had used anti-malarial drugs irregularly. The diagnostic performance characteristics of OptiMAL test are given in the Table.

\section{DISCUSSION}

The recently developed rapid malaria diagnostic test is an effective tool in the diagnosis of malarial infections. The world health community is in urgent need of rapid precise diagnostic tools to assist in controlling malaria, monitoring drug therapy, and reducing morbidity and mortality.

The OptiMAL test is an one-step coloured immunochromatographic assay which was designed as an in vitro diagnostic test for the detection of $P$. vivax and $P$. falciparum malaria in whole blood.

We investigated the diagnostic performance characteristics of the OptiMAL test. The diagnostic sensitivity, specificity, positive predictive value, negative predictive value and efficiency of the test were determined. The relationship between the parasite number and the diagnostic power of the

TABLE

Diagnostic performance characteristics of the OptiMAL test for the diagnosis of vivax malaria

\begin{tabular}{ccccccc}
\hline Parasites/ $\mu \mathrm{l}$ & $\begin{array}{c}\text { Samples } \\
\text { positive } \\
\text { by dipstick }\end{array}$ & $\begin{array}{c}\text { Samples } \\
\text { positive } \\
\text { by blood film }\end{array}$ & Sensitivity & Specificity & $\begin{array}{c}\text { (+)predictive } \\
\text { value }\end{array}$ & $\begin{array}{c}\text { (-)predictive } \\
\text { value }\end{array}$ \\
\hline $1-500$ & 42 & 46 & 0.91 & 1 & 1 & 0.96 \\
$501-1000$ & 5 & 5 & 1 & 1 & 1 & 1 \\
$1001-5000$ & 18 & 19 & 0.94 & 1 & 1 & 0.99 \\
$5001-10000$ & 10 & 10 & 1 & 1 & 1 & 1 \\
\hline Total & 76 & 81 & 0.93 & 1 & 1 & 0.95 \\
\hline
\end{tabular}


test was also evaluated. In addition, the number of parasites and the intensity (clear or pale) of the color of the positivity line which forms on the test strip were evaluated (Figure). It was determined that the intensity of the colour varied according to the parasitemia level, in the case of a low level of parasitemia, a pale positive line appeared whereas the color of the line was distinct when the level of parasitemia was high.

As it may be seen in the Table, 81 of the 190 patients who had malaria-like symptoms were diagnosed as having $P$. vivax malaria on the basis of thick-film examination and clinical findings. Seventy-six of these 81 individuals were positive according to the OptiMAL test. Five cases were found to be false negative with the OptiMAL test, four of which exhibited 1-500 parasites/ $\mu$, while one ap-

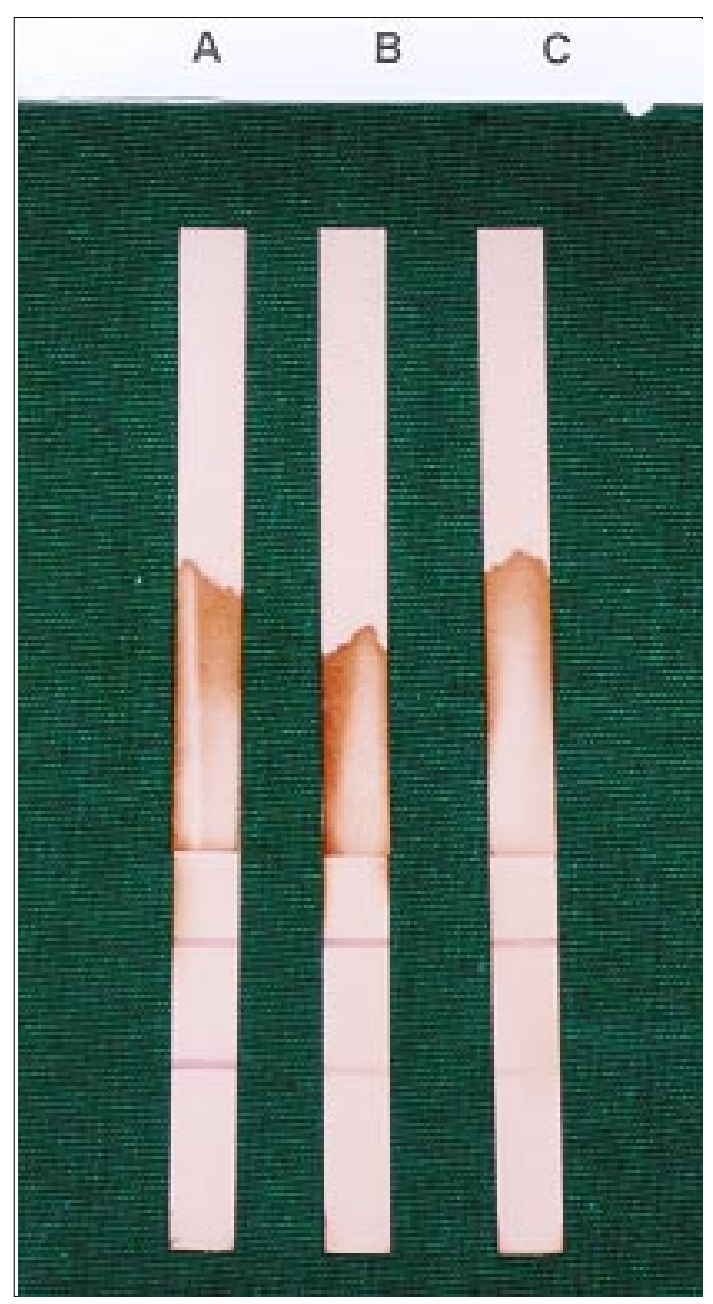

Three dip stick results which the intensity of the colour varied according to the parasitemia level, the color of the line was distinct when the level of parasitemia was high (A) whereas in the case of a low level of parasitemia, a pale positive line appeared (C, B). peared to contain 12000 parasites $/ \mathrm{ml}$. Three of the five false negative patients had used anti-malarial drugs irregularly.

The sensitivity of the OptiMAL test was decreased in cases of less than 500 parasites/ $\mu \mathrm{l}$, as has also been reported in other studies (Palmer et al. 1998). There was a discrepancy between the thick-film and OptiMAL tests in one case in which the parasite count was $2000 / \mu l$. This case was examined several times, but the result was unchanged. No reasonable explanation for this could be found. No false positive results were detected with the OptiMAL test. The specificity of the OptiMAL tests was $100 \%$ for all parasite counts and the positive predictive value was one.

The diagnosis of malaria relies on the microscopic examination of blood films. However, the ParaSight-F test for the specific detection of $P$. falciparum antigen has been developed, and this is $98 \%$ sensitive and $100 \%$ specific (Swift et al. 1993). It may be useful for quick diagnosis when microscopy is not available. Nevertheless, as it detects only the $P$. falciparum antigen, the identification of other plasmodium species is impossible when this test is used alone. However, an alternative test, the OptiMAL assay, has been developed, which is based on the detection of an abundant intracellular metabolic enzyme ( $\mathrm{pLDH}$ ) produced by viable malaria parasites. The $\mathrm{pLDH}$ is present in, parasite-infected red blood cells and is released from them. The OptiMAL ${ }^{\circledR}$ assay detects the pLDH enzyme with a series of monoclonal proteins. The differentiation of malaria species is based on antigenic differences between the pLDH isoforms.

Berchem et al. (1999) reported that the sensitivity of ICT testing is related to the rate of parasitemia. In cases where $50-500 / \mathrm{ml}$ parasitemia was detected, the sensitivity was $85 \%$. They also reported false results with ICT testing in whose thickblood films were negative and who had received anti-malarial treatment two or three times previously.

In a study carried out in Honduras, Palmer et al. (1998) using the OptiMAL test, were unable to identify 5 out of 79 malaria patients who tested positive for $P$. vivax with the thick-blood film method. They reported that the sensitivity of the OptiMAL test was $94-98 \%$, while the specificity of the test was $99-100 \%$. As it may be seen in the Table, the results of the present study are in agreement with these findings.

The fact that some of the malaria infections detected by blood films were not detected by the OptiMAL test may be because the OptiMAL test only detects pLDH which is expressed by living parasites. There are several possible explanations for discrepancies in the results obtained by blood- 
film examination and by the OptiMAL test, (i) insufficient detection of low parasitemia levels by OptiMAL; (ii) the fact that OptiMAL detects only live parasites producing pLDH; and (iii) the sequestration of parasites (Jelinek et al. 1999).

In conclusion, the rapid malarial diagnosis test, OptiMAL, is specific and efficient. This simple, rapid test can be used in the diagnosis of vivax malaria in the situations where specialized laboratory facilities and personnel are not available.

\section{REFERENCES}

Beadle C, Long GW, Weiss WR, McElroy PD, Maret SM, Oloo AJ, Hoffman SL 1994. Diagnosis of malaria by detection of Plasmodium falciparum HRP2 antigen with a rapid dipstick antigen-capture assay. Lancet 343: 564-568.

Berchem NN, Leke RF, Tietche F, Taylor DW 1999.

Evaluation of a rapid test for histidine rich protein 2 for diagnosis of Plasmodium falciparum infection in Cameroonian children. Trans $R$ Soc Trop Med Hyg 93: 46.

Garcia A, Kirimoama S, Marlborough D, Leafasia J, Rieckmann KH 1996. Immunochromatographic test for malaria diagnosis. Lancet 347: 1549.

Krogstad DJ 1996. Malaria as a reemerging disease.
Epidemiol Rev 18: 77-89.

Jelinek T, Grobusch PM, Schwenke S, Steild S, Sonnenburg von F, Nothdurft HD, Klein E, Löscher T 1999. Sensitivity and specificity dipstick test for rapid diagnosis of malaria in nonimmune travelers. Clin Microbiol 3: 721-723.

Palmer CK, Lindo JF, Klaskala WI, Quesada JA, Kaminsky R, Baum MK, Ager AL 1998. Evaluation of the OptiMal test for rapid diagnosis of Plasmodium vivax and Plasmodium falciparum Malaria. Clin Microbiol 1: 203-206.

Parra ME, Evans CB, Taylor D 1991. Identification of Plasmodium falciparum histidine-rich protein II in the plasma of humans with malaria. J Microbiol 29: 1629-1634.

Swift CJ, Premji Z, Minijas JN 1993. The rapid manual ParaSight-F test. A new diagnostic tool for Plasmodium falciparum infection. Trans $R$ Soc Trop Med Hyg 87: 646-648.

Tirasophon W, Rajkulchai P, Ponglikitmongkol M, Wilairat P, Boonsaeng V, Panyim S 1994. A highly sensitive, rapid, and simple polymerase chain reaction-based method to detyect human malaria (Plasmodium falciparum and Plasmodium vivax) in blood samples. Am J Trop Med Hyg 3: 308-311.

Warhurst DC, Williams JE 1996. Laboratory diagnosis of malaria. J Clin Pathol 49: 533-538. 